ESAIM: PROCEEDINGS, August 2010, Vol. 30, p. 15-33

D. Bresch, V. Calvez, E. Grenier, P. Vigneaux \& J.-F. Gerbeau, Editors

\title{
A MATHEMATICAL MODEL OF INFLAMMATION DURING ISCHEMIC STROKE
}

\author{
Cristiana Di Russo ${ }^{1}$, Jean-Baptiste Lagaert ${ }^{2}$, Guillemette Chapuisat ${ }^{3}$ and \\ MARIE-AimÉE Dronne ${ }^{4}$
}

\begin{abstract}
In this article we propose a model to describe the inflammatory process which occurs during ischemic stroke. First, an introduction to some basic concepts about the biological phenomenon is given. Then, a detailed derivation of the model and the numerical scheme used are presented. Finally, the studies of the model robustness and sensitivity are showed and some numerical results on the time and space evolution of the process are presented and discussed.

Résumé. Dans cet article, nous nous attachons à modéliser le processus inflammatoire lors d'un accident vasculaire cérébral ischémique. Tout d'abord, nous expliquons brièvement les bases biologiques de ce phénomène. Puis, nous décrivons les équations et les schémas numériques retenus pour le modéliser et l'implémenter. Nous présentons ensuite des études de robustesse et de sensibilité et montrons et discutons les premiers résultats de simulation obtenus.
\end{abstract}

\section{INTRODUCTION}

Strokes are the second worldwide death cause and the sixth source of handicap in the world [1,2]. They consist in a rapid developing loss of brain functions due to a disturbance in the cerebral blood flow. This can be due to ischemia ( $80 \%$ of stroke), when the lack of blood supply is caused by thrombosis or embolism, or due to hemorrhage. During a stroke, the affected area of the brain is unable to function any more, leading to trouble moving, walking, seeing, speaking or understanding. It is a medical emergency and can cause permanent neurological damage, complications, and death.

We focus our study on one of the pathophysiological mechanisms involved in ischemic stroke, the inflammatory process $[3,4]$. In a general setting, inflammation is a complex biological response of vascular tissues to harmful stimuli such as pathogens, damaged cells or irritants. During ischemic stroke, inflammation is triggered to eliminate the dead cells but can also lead to the death of some other cells. Our purpose is to obtain in silico experiments (i.e. simulations on a computer) to study and discuss the influence of the inflammation during stroke and to propose some possible therapeutic approaches.

\footnotetext{
1 Dipartimento di Matematica, Università degli Studi Roma Tre, Largo San Leonardo Murialdo 1, I-00146, Roma, Italy

${ }^{2}$ Université de Bordeaux, Institut de Mathématiques, INRIA Sud-Ouest MC2, 351 cours de la Libération, 33405 Talence Cedex, France

${ }^{3}$ LATP, UMR 6632, Université Paul Cézanne Aix-Marseille 3, avenue Escadrille Normandie-Niemen 13397 Marseille Cedex 20 , France

${ }^{4}$ Laboratoire de Biométrie et Biologie Evolutive, UMR 5558, Université Claude Bernard Lyon 1, 69000 Lyon, France
}

(C) EDP Sciences, SMAI 2010 
Ischemic stroke begins with the decrease of the cerebral blood flow which can drop below $10 \%$ of the normal blood flow. Cells around the occluded vessel begin to die and create what we call the infarcted core. Around this infarcted core, we can find the penumbra which is an area of moderate ischemia and which is able to recover thanks to reperfusion or therapeutic intervention. In these two ischemic areas, cells can die either through necrosis or through apoptosis. Necrosis occurs mainly in the infarcted core and occurs very early after the stroke onset. It leads to the rupture of cell membrane, the disintegration of intracellular organelles and the release of intracellular contents in the extracellular space. As a consequence, necrotic cells "pollute" the environment and damage the surrounding cells. On the contrary, apoptosis appears later (from 30 minutes up to 2 or 3 days after the stroke onset) and apoptotic death is much slower than necrosis as shown in Rupalla et al. [5]. Moreover, apoptosis occurs mainly in the penumbra and is not deleterious for the neighboring cells. We can also notice that, as indicated in [6] by Ankarcrona et al., apoptosis can lead to the inflammatory process, even if it was observed that inflammatory process is mainly induced by necrotic cells in the infarcted core. So, inflammation contribute to cell death by necrosis [7] and apoptosis [8,9].

During an ischemic stroke, the first phase of the inflammatory process consists in the activation of microglia. Microglia are the resident immune cells aimed at protecting brain cells. In ischemic conditions, microglia get activated: their shapes change, which increases their abilities to phagocytosis and production of cytokines and chemokines. Cytokines are proteins that trigger the accumulation of adhesion molecules on the vascular endothelium leading to the entrance of leukocytes (i.e immune cells circulating in the blood) into brain tissue. Among cytokines, chemokines induce chemotaxis in nearby responsive cells to attract leukocytes from the blood. Moreover, activated microglial cells are able to phagocytize necrotic and apoptotic cells as showed by Vilhardt in [10] and by Schilling et al. in [11]. But, during phagocytosis, microglia produce and release free radicals such as nitric oxide (NO) which is deleterious for the surrounding cells. Thus, microglia have both deleterious roles through the production of toxic substances and beneficial ones through the prevention of damage extension by phagocytosis and the production of trophic molecules and anti-inflammatory cytokines that can mediate neuroprotection and tissue repair [12].

The second phase of the inflammatory process consists in the infiltration of leukocytes in brain tissue. The leukocytes involved in this process are neutrophils and monocytes. The latter are called macrophages once they leave the blood to enter the tissue. Neutrophils infiltrate the tissue about 12 hours after the stroke onset and produce lots of deleterious substances (that are useful to slow down an infection for example but that are totally counterproductive in an ischemic stroke). Moreover, they are able to phagocytize small quantities of dead bodies and produce free radicals like NO [13]. Macrophages infiltrate the tissue later, about 24 hours after the stroke onset. Like microglia, they produce cytokines, chemokines and free radicals and they are able to phagocytize necrotic and apoptotic cells. These leukocytes enter cerebral tissue through their interactions with the adhesion molecules located on endothelium. These cells have a beneficial role by cleaning the infarcted core and allowing the tissue cicatrization but they can also increase the ischemic damage by producing free radicals and pro-inflammatory cytokines as presented by Hallenbeck and Dutka in [14]. So, as microglia, these cells have both beneficial and deleterious effects during the inflammatory process.

The two phases of inflammation influence both in a positive and a negative way the survival of neurons and glial cells. In this study, we are interested in understanding which influence dominates, depending on the situation. Our final aim is to understand if and how it is possible to control the positive and negative aspects of this biological process, which could be helpful for the development of new therapeutic strategies in ischemic stroke.

In the literature, there are several models of systemic inflammation. For example, the models proposed by Ibragimov et al. [15], Kumar et al. [16], Lauffenburger and Kennedy [17] and Reynolds et al. [18] describe the behavior only of blood cells. Instead Ladeby et al. in [19] and Lai and Todd [20] consider also glial cells. Adrian 
and Marshall in [21], Eldestein-Keshet and Spiros in [22], Gray and Brookmeyer in [23], Mentis et al. in [24] and Ridall et al. in [25] describe models for neurodegenerative diseases. These works are focused on the behavior of cell components at subcellular level of a single cell type. In our model, we considered the cell level and we modelled the behaviors of several types of cells (microglia, leukocytes, neurons and astrocytes). As a starting point, we considered the cell model proposed recently by T. Lekelov-Boissard et al. [26]. They proposed an ODEs model and took into account the two phases of inflammation: activation of microglia and infiltration of blood leukocytes. In their model, they studied the dynamics of the densities of cells dead by necrosis and apoptosis, and of living cells. Moreover they considered the proportion of activated and inactivated resident microglia and the proportion of neutrophils and macrophages in the tissue. They also introduced the release of pro-inflammatory molecules (like cytokines, chemokines and free radicals) by microglia and leukocytes and their phagocytic abilities. But this model is "phenomenological" and does not consider the space dimension. Using the methods proposed in [26], we introduced in the model the space dimension. More precisely, we introduced the diffusion and the chemotaxis of proteins and leukocytes.

In this study, we thus developed and analysed a new model of inflammation during ischemic stroke. The paper is organized as follows: in Section 1 we present the derivation of the model, the numerical schemes used to approximate the solutions of the system and we discuss about the parameters that we have introduced in the model. In Section 2 we report some numerical results: we present the evolution of the process, the study on the robustness and sensitivity of parameters and the study on the influence of initial size of the infarcted core. In the last Section, we summarize the results obtained and we propose possible improvements.

\section{The MATHEMATiCAL MODEL}

\subsection{The equations}

\subsubsection{Global overview}

In our model, we considered a macroscopic level with a cell population scale. Thus, we used ordinary and partial differential equations to describe inflammation. The model reproduces the inflammatory process during the first 72 hours of the stroke. Every function depends on the time $t$ and on the space $x$. Figure 1 represents the mechanisms included in our model.

The functions of the model are:

$H$ : density of healthy brain cells in relation to the usual total number of brain cells,

$N$ : density of necrotic cells in relation to the usual total number of brain cells,

$A_{s}$ : density of cells that have started the apoptosis process in relation to the usual total number of brain cells,

$A_{e}$ : density of cells that have ended the apoptosis process in relation to the usual total number of brain cells,

$M_{i}$ : density of inactivated microglia in relation to the usual total amount of microglia,

$M_{a}$ : density of activated microglia in relation to the usual total amount of microglia,

$L_{m}$ : density of macrophages in relation to the maximal number of macrophages that can occupy a point of the space,

$L_{n}$ : density of neutrophils in relation to the maximal number of neutrophils that can occupy a point of the space,

$[c y]$ : concentration of pro-inflammatory cytokines,

$[c h]$ : concentration of chemokines,

$\mathcal{M}_{\text {adh }}$ : density of adhesion molecules. 

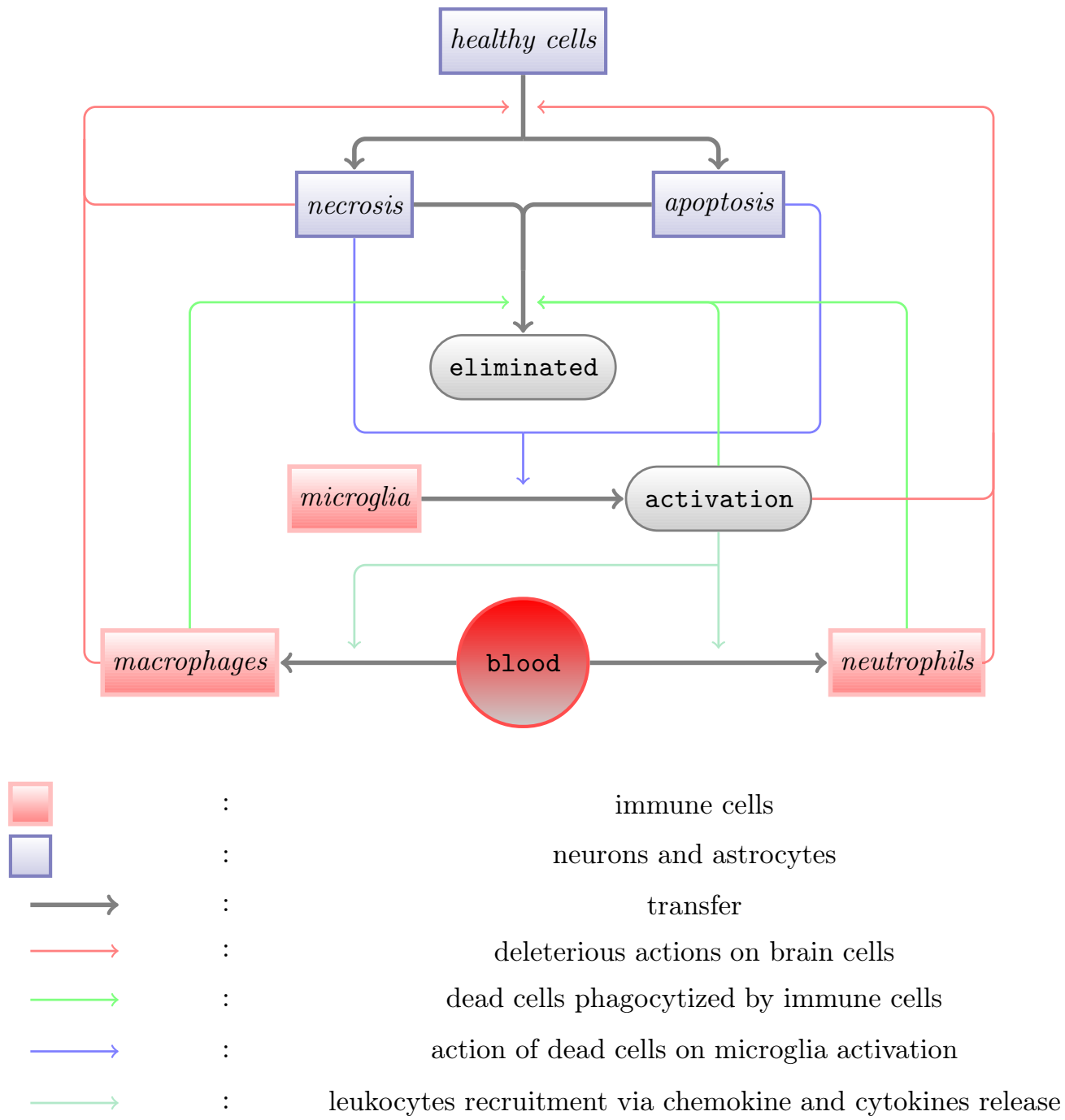

FiguRE 1. Connections between the different cells. 1) When an ischemic stroke occurs, neurons and glial cells die by necrosis or apoptosis. 2) These dead cells trigger the activation of the resident microglia and, due to the toxic substances in the environment, also to the death of other cells. 3) Activated microglia are able to phagocytize dead cells but they also produce cytokines (triggering the accumulation of adhesion molecules) and chemokines (attracting leukocytes). 4) Then, macrophages and neutrophils infiltrate brain tissue. These cells are able to phagocytize but they also produce toxic substances which are deleterious for healthy cells.

\subsubsection{Neurons and Astrocytes}

Neurons and glial cells such as astrocytes are resident brain cells which can be damaged during an ischemic stroke. We considered four different states for these cells: healthy cells, two types of dying cells (through apoptosis or through necrosis) and dead cells that have been eliminated from the tissue by phagocytosis. 
Cell death can occur either by necrosis or by apoptosis. These two different mechanisms have different roles, time scales and consequences in the inflammatory process. Thus, we modelled them separately. Moreover, to distinguish the early reversible phase of the apoptotic cascade from the late irreversible phase, we used two variables $A_{s}$ and $A_{e}$ for apoptotic cells. The cells beginning their apoptotic cascade are denoted $A_{s}$ and those ending their apoptotic cascade are denoted $A_{e}$.

The biological facts we wanted to reproduce are the following ones:

- Cells are mainly damaged by cytokines and other substances produced by neutrophils and by the deleterious substances released by the surrounding necrotic cells (we neglected the diffusion of the two last substances).

- When damage is important, cells die. Some of them die through necrosis and the other through apoptosis.

- Apoptosis is not instantaneous and needs a delay $t_{A}$.

- Microglial cells, macrophages and neutrophils phagocyte dead cells and eliminate them of the tissue.

Finally, since neurons and astrocytes do not have any mobility, we proposed the following laws:

$$
\begin{aligned}
\partial_{t} N & =p_{N} \mathcal{D} H-\mathcal{E} N \\
\partial_{t} A_{s} & =p_{A} \mathcal{D} H-p_{A} \mathcal{D}\left(.-t_{A}\right) H\left(.-t_{A}\right), \\
\partial_{t} A_{e} & =p_{A} \mathcal{D}\left(.-t_{A}\right) H\left(.-t_{A}\right)-\mathcal{E} A_{e}, \\
\partial_{t} H & =-\mathcal{D} H
\end{aligned}
$$

with $\mathcal{D}$ the dying cells density, $\mathcal{E}$ the density of cells which are phagocytizing, equal to:

$$
\begin{aligned}
\mathcal{D} & =\left[\left(p_{N,[c y]}[c y]+p_{N, L_{n}} L_{n}\left(N+A_{e}\right)+p_{N, N} N\right)-\mathcal{D}_{0}\right]^{+}, \\
\mathcal{E} & =e_{N, M_{a}} M_{a}+e_{N, L_{m}} L_{m}+e_{N, L_{n}} L_{n}+e_{N, M_{i}} M_{i} .
\end{aligned}
$$

In equations (1) and (2), $p_{N}$ and $p_{A}$ represent respectively the proportion of cells dying through necrosis or through apoptosis. Hence $p_{N}+p_{A}=1$.

\subsubsection{Microglia}

The microglial behavior is not very clear in spite of many experimental studies on these cells. In this model, we considered the following mechanisms:

- Apoptotic and/or necrotic cells in the tissue trigger the activation of microglia.

- In absence of any stimulus, microglia get progressively inactivated.

- During stroke, inactivated microglial cells multiply by mitosis. This process takes a long time (about 24 hours).

Also these cells do not have mobility, so their densities verify the following ODEs:

$$
\begin{aligned}
& \partial_{t} M_{a}=\left(c_{A} A_{e}+c_{N} N\right) M_{i}-\frac{M_{a}}{T_{M, 1}} \\
& \partial_{t} M_{i}=-\left(c_{A} A_{e}+c_{N} N\right) M_{i}+\frac{M_{a}}{T_{M, 1}}+c_{M_{i}} M_{i}\left(1-M_{i}\right) \mathbf{1}_{t>T_{M, 2}} .
\end{aligned}
$$

$\mathbf{1}_{t>T_{M, 2}}$ is a characteristic function hence is equal to 1 if $t>T_{M, 2}$ and equal to 0 otherwise.

\subsubsection{Leukocytes}

The immune blood cells are called leukocytes. They circulate in the non occluded vessels and enter the ischemic tissue from these vessels. So, in our model, we assume that there is no infiltration of these cells through the obstructed vessel. There are two cell species involved in this inflammation process: macrophages $\left(L_{m}\right)$ and neutrophils $\left(L_{n}\right)$. The biological assumptions are the following ones: 
- Leukocytes are recruited in blood vessels by chemical signals.

- They can enter brain tissue only through adhesion molecules.

- The crossing of the blood-brain barrier takes about 12 hours for leukocytes and 24 hours for macrophages.

- Once in the cerebral tissue, leukocytes are attracted by chemokines (by chemotaxis).

- In absence of any stimulus, leukocytes progressively leave the tissue.

We proposed the following equations:

$$
\begin{aligned}
\partial_{t} L_{m}-\overbrace{D_{L_{m}} \Delta L_{m}}^{\text {diffusion }}= & \overbrace{-\mu_{m} \nabla \cdot\left(L_{m}\left(1-L_{m}\right) \nabla[c h]\right)}^{\text {chemotaxis }} \\
& +\underbrace{c_{L_{m}} \mathcal{B}\left(.-T_{L_{m i n}}\right) \tilde{H}\left(L_{M}\right)}_{\text {carrying by blood }}-\underbrace{\frac{L_{m}}{T_{L_{m}}}}_{\text {natural decay }} \\
\partial_{t} L_{n}-D_{L_{n}} \Delta L_{n}= & -\mu_{n} \nabla \cdot\left(L_{n}\left(1-L_{n}\right) \nabla[c h]\right) \\
& +c_{L_{n}} \mathcal{B}\left(.-T_{L_{n i n}}\right) \tilde{H}\left(L_{M}\right)-\frac{L_{n}}{T_{L_{n}}}
\end{aligned}
$$

where $\mathcal{B}$ quantify the permeability of the blood vessel (in a healthy brain, the blood-brain barrier prevents the access to the brain) and $\tilde{H}(x)$ a regularization of the heavyside function to model a saturation effect:

$$
\begin{aligned}
\mathcal{B} & =\mathcal{M}_{\text {adh }} \\
\tilde{H}(x) & =\frac{1-\tanh R(0.75-x)}{2},
\end{aligned}
$$

where $\mathrm{R}=1000$.

Since the number of leukocytes in a point of brain is limited, we introduced a saturation in the chemotaxis term.

\subsubsection{Chemical species}

Let us denote by $[c y]$ and $[c h]$ the cytokine and the chemokine concentrations. In our model, we considered that cytokines were pro-inflammatory and their production, as for chemokines, was proportional to the number of macrophages and activated microglia. Moreover, these substances are progressively absorbed/degraded by the tissue.

As these chemical species diffuse in the tissue, we proposed the following equations:

$$
\begin{aligned}
\partial_{t}[c y]-\overbrace{D_{c y} \Delta[c y]}^{\text {diffusion }}=\overbrace{\left(p_{M_{a}, c y} M_{a}+p_{L_{m}, c y} L_{m}\right)\left(N+A_{e}\right)}^{\text {production by immune cells }}-\overbrace{e_{c y}[c y]}^{\text {natural decay }}, \\
\partial_{t}[c h]-D_{c h} \Delta[c h]=\left(p_{M_{a}, c h} M_{a}+p_{L_{m}, c h} L_{m}\right)\left(N+A_{e}\right)-e_{c h}[c h] .
\end{aligned},
$$

The parameters $p_{x, c y}, x=M_{a}$ or $L_{m}$, are production rates. Since we only considered pro-inflammatory cytokines in this model, it was necessary to transform these parameters into functions of time and cytokine concentration:

$$
p_{x, c y}=p_{x, c y, 0} \frac{1-[c y]}{1+t / t_{0}} .
$$

With this assumption, we considered that the anti-inflammatory cytokines were less secreted than the proinflammatory ones at the beginning of the inflammatory process, but that they were more secreted at the end of the process. This limits the effect of pro-inflammatory cytokines if they are too numerous.

Let us denote by $\mathcal{M}_{a d h}$ the density of adhesion molecules. From experimental data we know that cytokines trigger a production of adhesion molecules on the vessel endothelium. Moreover, without any cytokine, adhesion 
molecules progressively disappear. As these cells remain fixed in the blood vessel, their density follows an ordinary differential equation:

$$
\partial_{t} \mathcal{M}_{a d h}=[p_{\mathcal{M}_{a d h},[c y]} \overbrace{\left(1-\mathcal{M}_{a d h}\right)}^{\text {saturation }}[c y]-e_{\mathcal{M}_{a d h}} \mathcal{M}_{a d h}] \overbrace{\mathbf{1}_{\text {blood vessels }}^{\text {localisation }}}^{\text {nato }}
$$

\subsection{Summary}

We finally obtained a closed system of 13 equations. There are 7 ordinary differential equations to model fixed species :

$$
\begin{aligned}
\partial_{t} N & =p_{N} \mathcal{D} H-\mathcal{E} N, \\
\partial_{t} A_{s} & =p_{A} \mathcal{D} H-p_{A} \mathcal{D}\left(.-t_{A}\right) H\left(.-t_{A}\right), \\
\partial_{t} A_{e} & =p_{A} \mathcal{D}\left(.-t_{A}\right) H\left(.-t_{A}\right)-\mathcal{E} A_{e}, \\
\partial_{t} H & =-\mathcal{D} H \\
\partial_{t} M_{a} & =\left(c_{A} A_{e}+c_{N} N\right) M_{i}-\frac{M_{a}}{T_{M, 1}}, \\
\partial_{t} M_{i} & =-\left(c_{A} A_{e}+c_{N} N\right) M_{i}+\frac{M_{a}}{T_{M, 1}}+c_{M_{i}} M_{i}\left(1-M_{i}\right) \mathbf{1}_{t>T_{M, 2}}, \\
\partial_{t} \mathcal{M}_{a d h} & =\left[p_{\mathcal{M}_{a d h},[c y]}\left(1-\mathcal{M}_{a d h}\right)[c y]-e_{\mathcal{M}_{a d h}} \mathcal{M}_{a d h}\right] \mathbf{1}_{\text {blood vessels }} .
\end{aligned}
$$

And there are 4 reaction-diffusion equations to describe mobile species:

$$
\begin{aligned}
\partial_{t} L_{m}-D_{L_{m}} \Delta L_{m} & =-\mu_{L_{m}} \nabla \cdot\left(L_{m}\left(1-L_{m}\right) \nabla[c h]\right)+c_{L_{m}} \mathcal{M}_{a d h}\left(.-T_{L_{m i n}}\right) \tilde{H}\left(L_{M}\right)-\frac{L_{m}}{T_{L_{m}}}, \\
\partial_{t} L_{n}-D_{L_{n}} \Delta L_{n} & =-\mu_{L_{n}} \nabla \cdot\left(L_{n}\left(1-L_{n}\right) \nabla[c h]\right)+c_{L_{n}} \mathcal{M}_{a d h}\left(.-T_{L_{n i n}}\right) \tilde{H}\left(L_{M}\right)-\frac{L_{n}}{T_{L_{n}}} \\
\partial_{t}[c y]-D_{c y} \Delta[c y] & =p_{c y}\left(M_{a}+L_{m}\right)\left(N+A_{e}\right)-e_{c y}[c y], \\
\partial_{t}[c h]-D_{c h} \Delta[c h] & =p_{c h}\left(M_{a}+L_{m}\right)\left(N+A_{e}\right)-e_{c h}[c h] .
\end{aligned}
$$

33 parameters appear in our system. In the next section, we discuss how to determine the values of these parameters.

\subsection{Numerical Implementation}

\subsubsection{Discretisation}

There are three kinds of equations to solve: ordinary differential equation, basic reaction-diffusion and reaction-diffusion equations with a chemotaxis term. Thus we had to implement three different solvers: one for the diffusion terms, one for the reaction terms, and one for chemotaxis parts.

For all these equations we used Strang splitting. For instance, for a reaction-diffusion equation $\partial_{t} f-K \Delta f=P$ we proceeded as follows:

(1) we solve $\partial_{t} f=P$ for a half time step,

(2) then we solve $\partial_{t} f=K \Delta f$ for a complete time step,

(3) and finally we solve $\partial_{t} f=P$ for a half time step.

We used a finite volume discretization. It is classical for diffusion equations [27]: by integrating the diffusion term and using Stokes formula, we obtain an exact space discretization, and thus we easily transform the diffusion equation in a linear problem. As the diffusion coefficient is constant, there is no discretization difficulty to ensure flux continuity. 
The diffusion matrix being constant, we preferred to solve the associated linear problem by the inversion of the matrix by a LU method rather than an iterative method. By this way we only had to compute the inverse once for each diffusion equation to solve it at each time step.

The exponential reaction term $\partial_{t} f=\alpha f$ in the equations, was solved by recognizing, after multiplication by $\exp (t \alpha)$ the exact time derivate of $f \exp (t \alpha)$. This other source term was computed by using a simple Euler scheme.

\subsubsection{Chemotaxis solver}

The chemotaxis term was separated into a reaction term and a transport term:

$$
\begin{aligned}
\partial_{t} f & =-\mu \nabla \cdot(f(1-f) \nabla[c h]) \\
& =-(\mu(1-f) \nabla[c h]) \cdot \nabla f-(-(\mu \nabla \cdot((1-f) \nabla[c h])) f
\end{aligned}
$$

As usual, we used Strang splitting. Thus to solve equations (9) and (10), we had to solve:

(1) the reaction term for a half time step,

(2) the diffusion part for a half time step,

(3) the advection term for a complete time step,

(4) the reaction term for a half time step,

(5) the diffusion part for a half time step.

Solving two times the diffusion part was not too expensive because we only had to multiply by the inverse matrix, which is constant, depending only on the diffusion coefficient and we had already computed it in the initialization algorithm.

The advection part was solved by using an upwind scheme.

\subsection{Parameter adjustment}

One of the main problems in modeling and simulating the inflammatory process is that few parameter values are known. Some of these parameters have no biological or chemical or physical reality. Other have been measured during in vitro or in vivo experiments but, as the data come from various species, cells and experimental conditions, they cannot be mixed and used in a single model.

At first, we listed the parameters which could be determined by experimental data. The number of the other parameters had to be reduced to be able to perform a simulation: too many parameters can not be determined by inverse problem and the model would have too many freedom degrees. As a consequence, many sets of parameters would be able to reproduce the same behaviors. For this reason we could not attempt to fit them by inverse problem method. Moreover, our model would not stay pertinent anymore: as the space of mathematical solution of our differential system would be too high, we would not be able to distinguish "biological" solution to unreasonable solution. We explain - and justify - in 1.4.2 the assumptions done to reduce the number of unknown parameters.

As there were still unknown parameters after these simplifications, we fitted them by reproducing some behaviors which had been observed in in vitro or in vivo experiments. We have determined some "rules" about the behaviors of the different components involved in the biological phenomenon based on [13,28,29]. We chose the values of our parameters in order that the model could respect these rules.

\subsubsection{Parameter values determined on biological bases}

In this model, some values of the parameters are well known.

- Experimental studies have shown that there is a duplication of microglial store about 24 hours after the stroke onset, and this increase of microglial amount lasts several days [30]. It is not possible to know exactly when this increase begins, just after the stroke onset or just before 24 hours. So we assumed that the increase of microglial cells began about 18 hours after the stroke onset $\left(T_{M, 2}=18 \mathrm{~h}\right)$ with a 
rapid growth in the first hours.

- Garcia et al. in [13] observed that, in experiments performed on rats that underwent permanent ischemia, neutrophils infiltrate the tissue after 12 hours. Macrophages infiltrate the tissue later, after 24 hours. So, in our model, we assumed the following delay times: $T_{L_{n i n}}=12 \mathrm{~h}$ and $T_{L_{\text {min }}}=24 \mathrm{~h}$.

- in vitro studies of leukocytes have shown that macrophages can remain in brain tissue for 4 to 5 days while neutrophils remain only few hours in brain tissue [11,13]. Hence we fixed $T_{L_{m}}=90 \mathrm{~h}$ and $T_{L_{n}}=$ $3 \mathrm{~h}$.

\subsubsection{Parameter values arbitrarily determined}

Activated microglia and macrophages have been observed to have the same behaviors and the same features in brain tissue, which make them difficult to distinguish. So we assumed that their abilities of phagocytosis were the same $\left(e_{N, L_{m}}=e_{N, M_{a}}\right)$ and that their production rates of chemokines - respectively cytokines - were also the same $\left(p_{M_{a}, c y, 0}=p_{L_{m}, c y, 0}\right.$ and $\left.p_{M_{a}, c y}=p_{L_{m}, c h}\right)$. Neutrophils and inactivated microglial cells have also phagocytic activities but these activities are lower than those of macrophages and activated microglia. For simplicity, we assumed that $e_{N, L_{n}}=e_{N, M_{i}}$. Thus we obtained:

$$
\mathcal{E}=e_{N, 1}\left(M_{a}+L_{m}\right)+e_{N, 2}\left(L_{n}+M_{i}\right)
$$

Moreover we fixed arbitrarily that

$$
e_{N, 2}=\frac{e_{N, 1}}{4}
$$

The threshold of cell resistance to toxicity $\mathcal{D}_{0}$ was also fixed arbitrarily. This threshold is compulsory so that the resting state is stable but the quantification of damage is arbitrary. Thus we fixed $\mathcal{D}_{0}=0.02$. Moreover, we assumed that the diffusion coefficients of macrophages and neutrophils were the same $\left(D_{L_{m}}=D_{L_{n}}\right)$, that the diffusion coefficients of chemokines and cytokines were the same $\left(D_{c h}=D_{c y}\right)$, that the activations of microglia by necrotic or apoptotic cells were the same $\left(c_{A}=c_{N}\right)$ and finally that half of the cells died through necrosis $\left(p_{N}=p_{A}=0.5\right)$.

\subsubsection{Determination of the other parameter values}

With the simplifications established above, there are still 19 parameter values to determine. We fixed the values of these parameters in order to check the following biological assertions:

- The number of microglial cells doubles in 24 hours [30].

- The concentration of cytokines reaches its peak after 12 hours [31]. Cytokines and chemokines are degradated and gradually eliminated.

- The density of neutrophils decreases after 48 hours [13].

- The density of macrophages does not decrease in the first $72 \mathrm{~h}$ [13].

- The cytokine concentration reaches its peak in $12 \mathrm{~h} \mathrm{[32].}$

In the following, the set of assertions listed above will be called the basis of rules. The parameter values that meet this basis of rules are listed together to the others values in table 1 . This set of parameter values is called reference set in the following sections.

\section{RESUlts}

\subsection{Simulation of inflammation during an ischemic stroke}

We present here in silico experiments that represent an inflammatory process which occurs during 72 hours of a typical ischemic stroke. The infarcted core after 30 minutes of ischemia is a disc of radius $5.5 \mathrm{~mm}$ composed only of necrotic cells. In our simulations we used the reference set of parameter values. Figure 2 presents the 


\begin{tabular}{|c|c|c|}
\hline Parameter & & Value in the reference set \\
\hline$t_{A}$ & duration of apoptotic process & 12 \\
\hline$T_{M, 1}$ & characteristic time of microglia desactivation & 60 \\
\hline $\bar{T} T_{M, 2}$ & characteristic time of microglia duplication & 18 \\
\hline$T_{L_{\min }}$ & characteristic time of macrophages recruitment & 24 \\
\hline$T_{L_{n i n}}$ & characteristic time of neutrophils recruitment & 12 \\
\hline$T_{L_{m}}$ & characteristic time of macrophages degradation & 90 \\
\hline$T_{L_{n}}$ & characteristic time of neutrophils degradation & 3 \\
\hline$\overline{\overline{\mathcal{D}_{0}}}$ & threshold of cells resistance to toxicity & 0.02 \\
\hline$p_{N}$ & proportion of cells dead by necrosis & 0.5 \\
\hline$e_{N, 2}$ & elimination by neutrophils and inactivated microglia & 0.0125 \\
\hline$c_{N}$ & microglia activation by necrotic cells & 0.06 \\
\hline$\overline{D_{L_{n}}}$ & neutrophils diffusion coefficient & 1.5 \\
\hline$D_{c y}$ & cytokines diffusion coefficient & 0.2 \\
\hline$\overline{p p_{N,[c y]}}$ & cytokines toxicity & $\overline{0.1}$ \\
\hline$p_{N, L_{n}}$ & neutrophils toxicity & 0.4 \\
\hline$p_{N, N}$ & necrotic cells toxicity & 0.05 \\
\hline$e_{N, 1}$ & elimination by macrophages and activated microglia & 0.05 \\
\hline$c_{A}$ & microglia activation by apoptotic cells & 0.06 \\
\hline$c_{M_{i}}$ & mitosis rate of microglia & 0.38 \\
\hline$p_{\mathcal{M}_{a d h},[c y]}$ & adhesion molecules recruitment by cytokines & 5 \\
\hline$e_{\mathcal{M}_{\text {adh }}}$ & elimination rates of adhesion molecules & 0.1 \\
\hline$t_{0}$ & charac. time of balance between pro/contra infla. cytokines & $72 / 6$ \\
\hline$p_{c y, 0}$ & cytokines production rate & 10 \\
\hline$e_{c y}$ & cytokines elimination rate & 0.1 \\
\hline$p_{c h}$ & chemokines production rate & 4.5 \\
\hline$e_{c h}$ & chemokines elimination rate & 0.18 \\
\hline$c_{L_{m}}$ & macrophages recruitment rate & 24 \\
\hline$c_{L_{n}}$ & neutrophils recruitment rate & 28 \\
\hline$\mu_{m}$ & macrophages chemotaxis coefficient & 0.15 \\
\hline$\mu_{n}$ & neutrophils chemotaxis coefficient & 0.3 \\
\hline$D_{L_{m}}$ & macrophages diffusion coefficient & 1.5 \\
\hline$D_{c h}$ & chemokines diffusion coefficient & 0.2 \\
\hline
\end{tabular}

TABLE 1. Reference set of parameter values: at the beginning there are the values determined on biological bases, then values arbitrary determined and, at the end, values determined in accordance to the basis of rules.

numerical results obtained with a simulation over 72 hours. As required by the determination of parameter values, these results reproduce the basis of rules of section 1.4.3.

In figure 2(a), we can notice different behaviors related to the death process. As a matter of fact, there is a constant decrease of necrotic cells while, for apoptotic cells, there is first a growth and then a slower decrease due to the different elimination times of phagocytosis.

Figure 2(c) shows the dynamics of activated microglia and of all the microglial cells (activated and inactivated). We can notice a progressive activation of these resident immune cells that lasts for the whole time of simulation. On the contrary, we can observe a brutal increase of activated microglia due to its duplication.

In figure 2(d), we can observe a similar behavior of macrophages and neutrophils with a delay due to different 


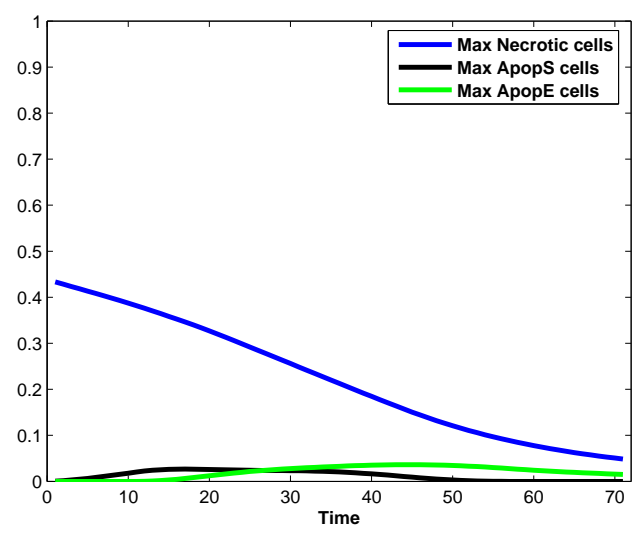

(a) Necrosis and apoptosis.

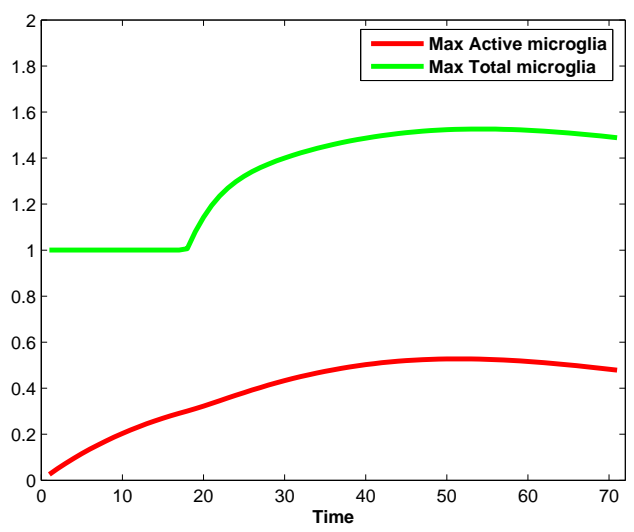

(c) Activated microglia and total microglial cells.

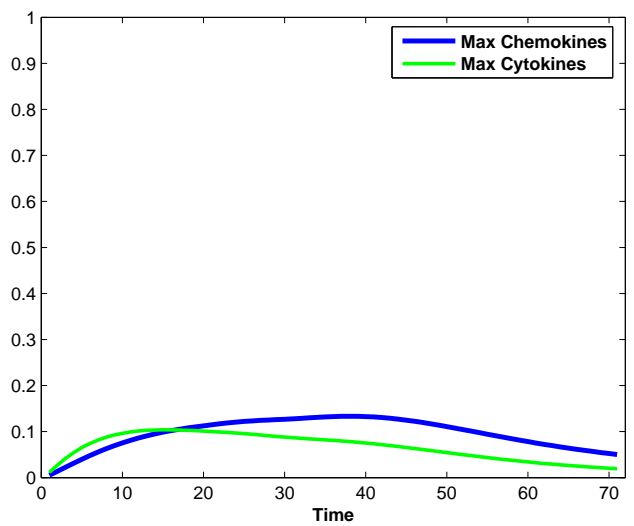

(e) Cytokines and chemokines

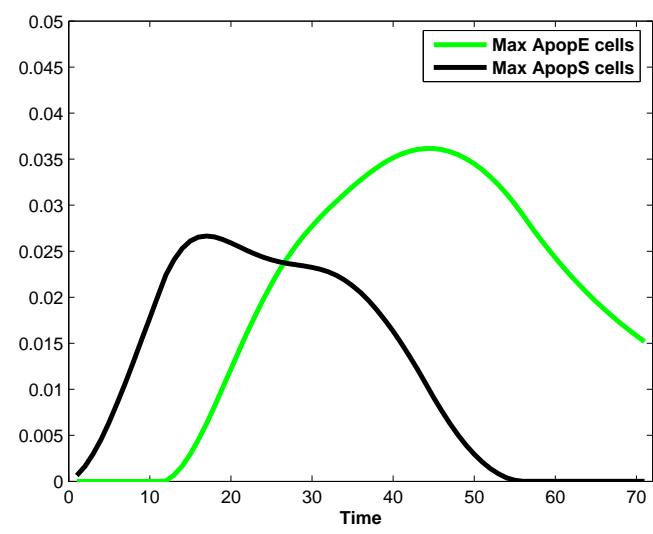

(b) Apoptosis (bigger scale).

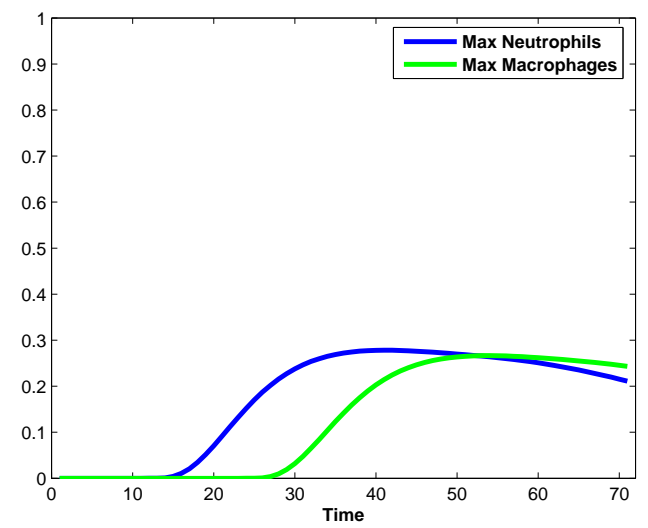

(d) Macrophages and neutrophils.

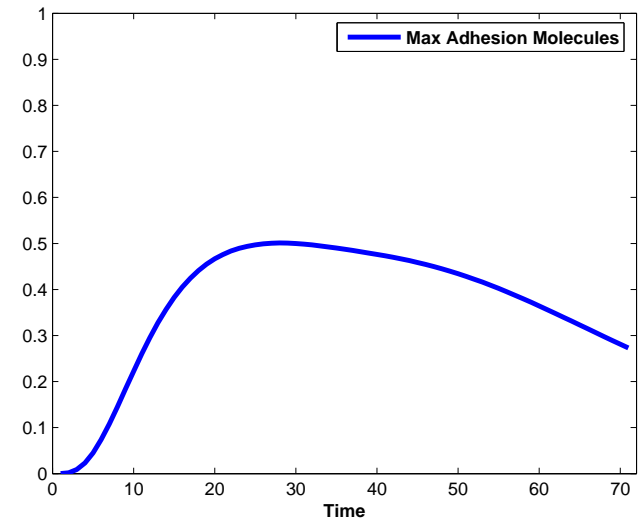

(f) Adhesion molecules

Figure 2. Time evolution of the maximum over space variable of various functions of the model. 
ESAIM: PROCEEDINGS

times of entrance into the tissue. We can also notice that, at the end of the simulation, when the dead bodies have been eliminated, leukocytes begin to decrease in brain tissue.

Figure 2(e) shows that the dynamics of the maximal concentration of cytokines is quite different from the dynamics of the maximal concentration of chemokines. We can see that the concentration of cytokines rapidly increases and slowly decreases whereas the concentration of chemokines slowly increases and decreases.

Figure 2(f) describes a rapid increase of adhesion molecules during the first 20 hours of the stroke. Then, we observe a stable level in the following 30 hours and, at the end of the process, we can see a slow decrease of this density.

\subsection{Robustness and Sensitivity of parameters.}

Our model includes many parameters and their values were chosen following a basis of rules as indicated in 1.4. We determined a reference set of parameter values and, before exploring the parameter space, we studied the influence of these parameters on the model. Thus we studied the robustness and the sensitivity of the model to these parameter values.

\subsubsection{Robustness}

In this part we wanted to check if the model with different values of parameters would still meet the basis of rules established in section 1.4.3. Hence we changed the value of one parameter keeping all the other parameter values unchanged. Then we solved the equations and checked if the basis of rules was still met. Results are summed up in table 2 .

We can sort the parameters in three classes:

- the parameters that have little influence on the model results $\left(T_{L_{n}}, T_{L_{m}}, T_{L_{m i n}}, T_{L_{n i n}}, c_{A}, c_{N}, t_{A}, T_{M, 1}\right.$, $\left.T_{M, 2}, t_{0}, D_{c h}, D_{c y}, p_{\mathcal{M}_{a d h}, c y}, C_{M_{i}}, p_{c h}\right)$,

- the parameters that have a moderate influence on the model results $\left(e_{c h}, c_{L_{m}}, c_{L_{n}}, \mu_{m}, D_{L_{n}}, D_{L_{m}}, D_{0}\right.$, $\left.p_{c y, 0}, e_{c h}, p_{N, c y}, p_{N, L_{n}}, p_{N, N}\right)$,

- The parameters that have a strong influence on the model results $\left(\mu_{n}, e_{N, 1}, e_{N, 2}, e_{\mathcal{M}_{\text {add }}}\right.$.).

We can notice that, even if we highly change the values of some parameters $\left(e_{c h}, c_{L_{m}}, c_{L_{n}}, \mu_{m}, D_{L_{n}}\right.$ and $D_{L_{m}}$ ), we obtain quite stable results. Therefore they affect mainly the related cells but do not influence the whole process. On the contrary, the interval range found for the neutrophils chemotaxis coefficient $\mu_{n}$ is $[0.03 ; 0.75]$ and its bigger modification brings to significant changes in the simulated results. Concerning parameters related to time, such as $T_{L_{n}}, T_{L_{m}}, T_{L_{m i n}}$ and $T_{L_{n i n}}$, we can note that changes in their values lead to negligible consequences in the simulated process.

\subsubsection{Sensitivity}

In this section, we studied the sensitivity of the model results to the parameter values. It is important to check this point before using the model for in silico experiments in order to be aware of the limitations due to parameter values used in the simulations. The sensitivity study can also give information for the further exploration of the parameter space.

To study the sensitivity, we considered six outputs of the model: the final dead area, the final density of macrophages, neutrophils, inactivated and activated microglia, cytokines, chemokines and adhesion molecules.

And we proceeded as follows: if $s\left(p_{1}, p_{2}, \cdots, p_{n}\right)$ is one of the chosen outputs obtained with the parameter values $\left\{p_{1}, p_{2}, \cdots, p_{n}\right\}$, the sensitivity of this output for example to the parameter $p_{1}$ is given by:

$$
S=\left(\frac{s\left(p_{1}+\varepsilon, p_{2}, \cdots, p_{n}\right)-s\left(p_{1}, p_{2} \cdots, p_{n}\right)}{s\left(p_{1}, p_{2} \cdots, p_{n}\right)}\right) /\left(\frac{\varepsilon}{p_{1}}\right) .
$$

In our study, we considered a change $\varepsilon$ equal to $\varepsilon=5 \%$ of the parameter.

The sensitivity of the size of the final dead area is small (equal to $4.10^{-4}$ ) for all the parameters except for the diffusion coefficient of the neutrophils $D_{L_{n}}$ where the sensitivity is null. Similarly, the sensitivity of the final 


\begin{tabular}{|c|c|c|}
\hline Parameter & Value in the reference set & Range of values where the rules are met \\
\hline$\overline{t_{A}}$ & $\overline{12}$ & $\pm 50 \%$ \\
\hline$T_{M, 1}$ & 60 & $\pm 50 \%$ \\
\hline$T_{M, 2}$ & 18 & {$[-90 \% ;+250 \%]$} \\
\hline$T_{L_{\min }}$ & 24 & {$[-95 \% ;+50 \%]$} \\
\hline$T_{L_{n i n}}$ & 12 & {$[-90 \% ;+300 \%]$} \\
\hline$T_{L_{m}}$ & 90 & {$[-97 \% ;+100 \%]$} \\
\hline$T_{L_{n}}$ & 3 & {$[-66 \% ;+3000 \%]$} \\
\hline$\overline{\overline{\mathcal{D}_{0}}}$ & $\overline{0.02}$ & {$[-20 \% ;+13 \%]$} \\
\hline$p_{N}$ & 0.5 & $\pm 10 \%$ \\
\hline$e_{N, 2}$ & 0.0125 & {$[-10 \% ;+20 \%]$} \\
\hline$c_{N}$ & 0.06 & $\pm 50 \%$ \\
\hline$\overline{D_{L_{n}}}$ & 1.5 & {$[-50 \% ;+3000 \%]$} \\
\hline$D_{c y}$ & 0.2 & {$[-50 \% ;+600 \%]$} \\
\hline$\overline{p_{N,[c y]}}$ & 0.1 & {$[-50 \% ;+30 \%]$} \\
\hline$p_{N, L_{n}}$ & 0.4 & {$[-50 \% ;+25 \%]$} \\
\hline$p_{N, N}$ & 0.05 & {$[-20 \% ;+120 \%]$} \\
\hline$e_{N, 1}$ & 0.05 & {$[-15 \% ;+25 \%]$} \\
\hline$c_{A}$ & 0.06 & $\pm 66 \%$ \\
\hline$c_{M_{i}}$ & 0.38 & {$[-95 \% ;+200 \%]$} \\
\hline$p_{\mathcal{M}_{a d h},[c y]}$ & 5 & {$[-50 \% ;+100 \%]$} \\
\hline$e_{\mathcal{M}_{a d h}}$ & 0.1 & {$[-50 \% ;+650 \%]$} \\
\hline$t_{0}$ & $72 / 6$ & {$[-50 \% ;+150 \%]$} \\
\hline$\overline{p_{c y, 0}}$ & 10 & $\pm 50 \%$ \\
\hline$e_{c y}$ & 0.1 & {$[-90 \% ;+400 \%]$} \\
\hline$p_{c h}$ & 4.5 & {$[-78 \% ;+100 \%]$} \\
\hline$e_{c h}$ & 0.18 & {$[-94 \% ;+200 \%]$} \\
\hline$c_{L_{m}}$ & 24 & {$[-84 \% ;+150 \%]$} \\
\hline$c_{L_{n}}$ & 28 & {$[-92 \% ;+115 \%]$} \\
\hline$\mu_{m}$ & 0.15 & {$[-80 \% ;+33 \%]$} \\
\hline$\mu_{n}$ & 0.3 & {$[-90 \% ;+150 \%]$} \\
\hline$\overline{D_{L_{m}}}$ & 1.5 & {$[-84 \% ;+3000 \%]$} \\
\hline$\overline{D_{c h}}$ & 0.2 & {$[-50 \% ;+2500 \%]$} \\
\hline
\end{tabular}

TABle 2. Robustness study. Range of values for each parameter where the model still meets the basis of rules described in 1.4.3. Biologically fixed parameters at first, arbitrary fixed at second and the others parameter at the end.

total density of inactivated microglia is null for all the parameters. The results concerning the sensitivities of the other output functions are presented in table 3 . We made other simulations with a smaller change $(\varepsilon=0.5 \%$ of the value of parameter) but the sensitivity results were similar.

Most parameters appear to have small influences on the main outputs of the model. As a consequence, we can introduce some small variations on the parameter values without changing the simulation results. But few parameters $\left(D_{0}, p_{N,[c y]}, p_{N, N}, e_{N, 1}, e_{N, 2}, p_{N}, c_{N}, T_{M, 1}, p_{\mathcal{M}_{a d h},[c y]}, e_{\mathcal{M}_{a d h}}, t_{0}, e_{c y}, p_{c y, 0}\right)$ have a strong influence on the model outputs.

We can also notice that the proportion of cells dying by necrosis and apoptosis (represented by parameters $\left.p_{N}\right)$ is very significant in the model, because a little change of its value leads to important changes in the final 


\begin{tabular}{|c|c|c|c|c|c|c|}
\hline Parameter & Num. of $L_{m}$ & Num. of $L_{n}$ & Num. of $M_{a}$ & Mass of $[c y]$ & Mass of $[c h]$ & Mass of $\mathcal{M}_{\text {adh }}$ \\
\hline \hline reference values & 1.4707 & 1.0184 & 0.1514 & 0.0978 & 0.1876 & 0.0272 \\
\hline \hline$t_{A}$ & 0 & 0 & 0 & 0 & 0 & 0 \\
\hline$T_{M, 1}$ & + & + & ++ & + & + & + \\
\hline$T_{M, 2}$ & 0 & 0 & 0 & 0 & 0 & 0 \\
\hline$T_{L_{m i n}}$ & 0 & 0 & 0 & 0 & 0 & 0 \\
\hline$T_{L_{n i n}}$ & 0 & + & 0 & + & + & 0 \\
\hline$T_{L_{m}}$ & 0 & 0 & 0 & 0 & 0 & 0 \\
\hline$T_{L_{n}}$ & 0 & + & 0 & 0 & 0 & 0 \\
\hline \hline $\mathcal{D}_{0}$ & ++ & ++ & + & +++ & +++ & ++ \\
\hline$p_{N}$ & +++ & +++ & +++ & +++ & +++ & +++ \\
\hline$e_{N, 2}$ & + & ++ & ++ & +++ & +++ & ++ \\
\hline$c_{N}$ & ++ & ++ & ++ & + & + & + \\
\hline$D_{L_{n}}$ & 0 & 0 & 0 & 0 & 0 & 0 \\
\hline$D_{c y}$ & + & ++ & + & 0 & + & 0 \\
\hline \hline$p_{N,[c y]}$ & + & + & + & ++ & ++ & ++ \\
\hline$p_{N, L_{n}}$ & 0 & 0 & 0 & + & + & 0 \\
\hline$p_{N, N}$ & ++ & ++ & + & +++ & +++ & ++ \\
\hline$e_{N, 1}$ & + & ++ & + & +++ & +++ & ++ \\
\hline$c_{A}$ & 0 & 0 & 0 & 0 & 0 & 0 \\
\hline$c_{M_{i}}$ & 0 & 0 & 0 & 0 & 0 & 0 \\
\hline$p_{\mathcal{M}_{a d h},[c y]}$ & ++ & ++ & 0 & + & + & ++ \\
\hline$e_{\mathcal{M}_{a d h}}$ & ++ & ++ & 0 & + & + & +++ \\
\hline$t_{0}$ & ++ & +++ & 0 & +++ & ++ & ++ \\
\hline$p_{c y, 0}$ & ++ & +++ & + & +++ & ++ & +++ \\
\hline$e_{c y}$ & ++ & ++ & 0 & ++ & + & ++ \\
\hline$p_{c h}$ & 0 & 0 & 0 & 0 & +++ & 0 \\
\hline$e_{c h}$ & 0 & 0 & 0 & 0 & ++ & 0 \\
\hline$c_{L_{m}}$ & +++ & 0 & 0 & + & + & + \\
\hline$c_{L_{n}}$ & 0 & +++ & 0 & + & + & 0 \\
\hline$\mu_{m}$ & 0 & 0 & 0 & 0 & 0 & 0 \\
\hline$\mu_{n}$ & 0 & 0 & 0 & 0 & 0 & 0 \\
\hline$D_{L_{m}}$ & +++ & 0 & 0 & 0 & 0 & 0 \\
\hline$D_{c h}$ & 0 & 0 & 0 & + & + & + \\
\hline$T_{A}$ & + & + & + & + & + \\
\hline
\end{tabular}

TABLE 3. Sensitivity study with $\varepsilon=5 \%$ of the parameter value in the reference set. In each row, you find a symbol which indicates the value of $S$ (with respect to each parameter) regarding the output written on the first line. Here " 0 " indicates that a parameter influences not at all the corresponding output variable, " + " that it influences a little, " + + that it influences moderatly and finally " +++ " indicates that the paramenter influences strongly the corresponding output. The thresholds chosen are the following $0 \leq 0<0.1,0.1 \leq+<0.4$, $0.4 \leq++<0.9$ and $+++\geq 0.9$.

quantities of leukocytes, microglia, cytokines, chemokines and adhesion molecules.

Concerning the final number of macrophages, we can observe that this quantity is sensible to several parameters such as $p_{\mathcal{M}_{a d h},[c y]}$ the production rate of adhesion molecules, $e_{\mathcal{M}_{a d h}}$ the natural decay of adhesion 
molecules, $p_{c y, 0}$ the production rate of cytokines by macrophages and microglia, $c_{L_{m}}$ a measure of the quantity of macrophages that filter into the tissue and $D_{L_{m}}$ the diffusion coefficient of these leukocytes. The final density of neutrophils has a similar response. It is sensitive to small changes in parameters related to adhesion molecules, and of course to the parameter $c_{L_{n}}$ which quantifies the neutrophils that infilter the tissue. It is interesting to notice that a small variation of the diffusion coefficient $D_{L_{n}}$ does not really influence them. Concerning activated microglia, we can note that they react also to small changes in the values of $c_{N}$, their activation rate by necrotic cells, and of $T_{M, 1}$, the characteristic time of microglia desactivation. For proteins, cytokines and chemokines, we notice similar behaviors. As a matter of fact, they react to small changes of the following parameters: $\mathcal{D}_{0}$, a threshold of damage of living cells, $p_{N, N}$ that estimates the effects of necrotic cells on dead cells elimination, and $e_{N, 1}, e_{N, 2}$ that are the elimination rates of dead cells by phagocytosis respectively of macrophages, activated microglia and inactivated microglia and neutrophils. These proteins are also affected by changes in their production rates, $t_{0}, p_{c y, 0}, p_{c h}$, and natural decay $e_{c y}, e_{c h}$.

Finally, we can note that the density of adhesion molecules is mainly sensitive to changes in their production

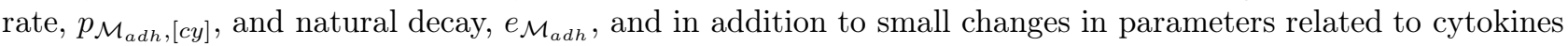
$t_{0}, p_{c y, 0}$ and $e_{c y}$.

\subsection{Influence of the size of the initial infarct}

We then used this model to carry out in silico experiments in order to explore the beneficial and/or deleterious effects of inflammation during stroke depending on the size of the initial infarct.
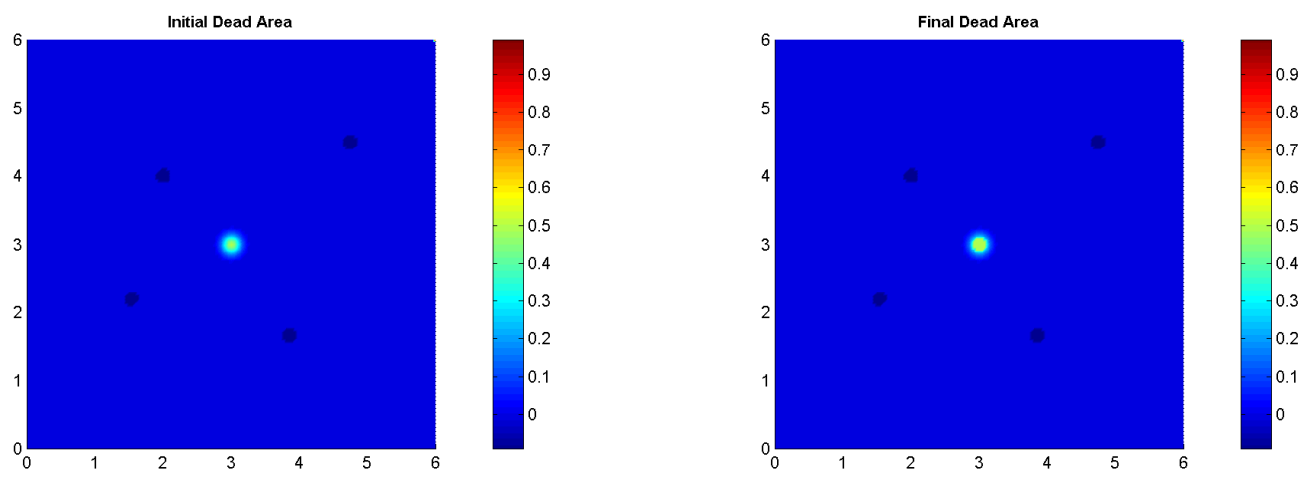

FIGURE 3. Initial and final dead areas obtained with an initial small size of the infarct.

Figures 3, 4 and 5 show the influence of initial dimension of the infarct in the development of the inflammatory process. We can observe that the aggravation due to inflammation is not a linear function of the initial size of the stroke. Therefore even if we had considered an initial infarct twice smaller (or twice bigger) than the standard stroke used in the previous simulations, the final size of the dead area would not have been twice smaller (or twice bigger) than the final area previously obtained. We can note that for small and medium initial data there is not an increase of the dead area, but, in the case of big size of the infarct, figure 5 , we have a significant increment of $235 \%$. In this case the initial dead area is 3.5911 , while the final dead area is 12.0504 , which correspond to $335 \%$ of the initial area. 

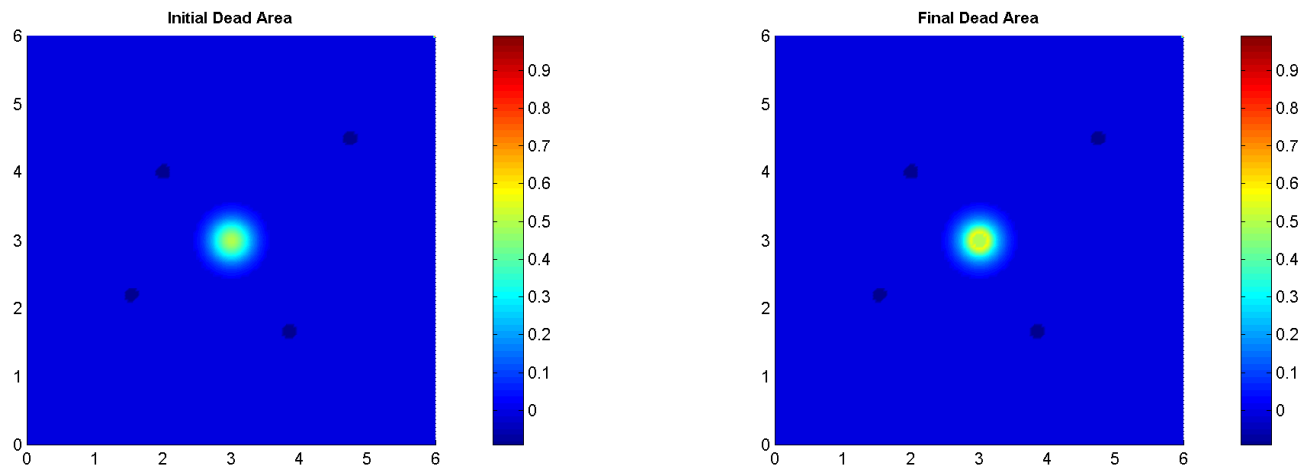

FiguRE 4. Initial and final dead areas obtained with an initial medium size of the infarct.
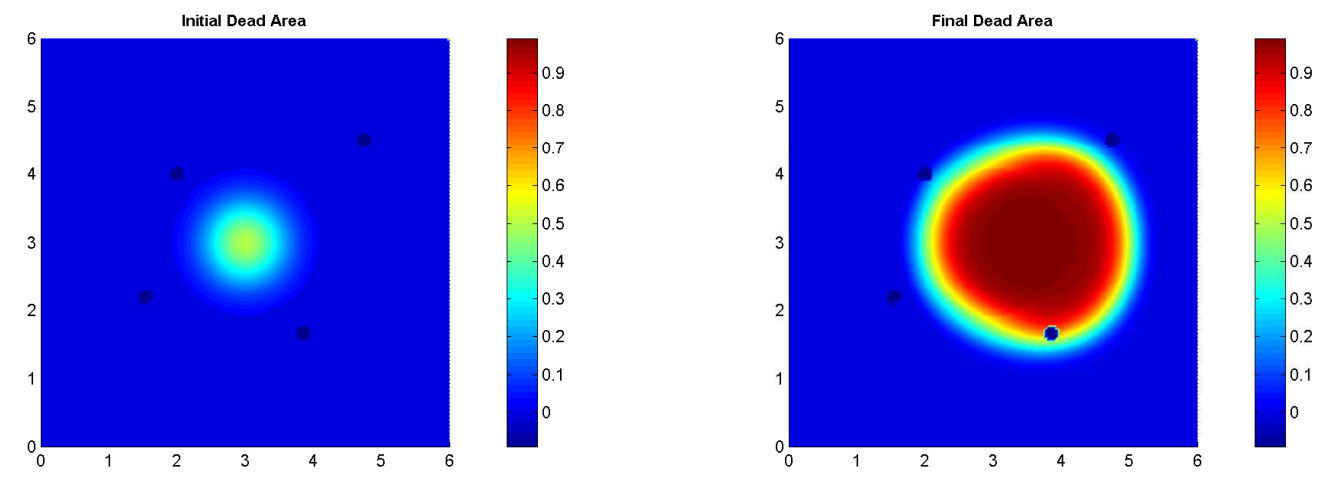

Figure 5. Initial and final dead areas obtained with an initial large size of the infarct.

\section{Discussion}

In this study, we built a model based on a set of ordinary and partial differential equations to represent the biological phenomena involved in the inflammatory process during an ischemic stroke. In our model, we considered different types of cells and of chemical substances. Therefore we represented the behavior of healthy, necrotic and apoptotic cells, and of immune cells like microglia, neutrophils, macrophages. Moreover we have included the cytokines, chemokines and adhesion molecules. The most important feature of the model is its spatial dimension, which allows to reproduce some mechanisms such as the diffusion of proteins and the recruitment of leukocytes by chemotaxis.

The model includes many parameters and one of the main problems was to determine the values of these parameters. We fixed these values with different methods (as explained in 1.4). Some parameters were determined by fitting the results of the model to real data coming from experiments on rats that underwent permanent ischemia [13], other parameters thanks to biological knowledge and the remaining parameters in order not to disturb the system. It was possible to find a set of parameter values that allows the model to respect the rules detailed in section 1.4.3. Thus, with this reference set of parameter values, we could obtain realistic simulations of the biological phenomenon. However a further study of the parameter space should be made to check if the qualitative behavior of the model is completely determined by those rules or if we have to complete them. 
In the robustness study (2.2.1), we mainly obtained narrow ranges of parameter values, which guarantees a quite stable behavior of the whole system. Another significant aspect underlined by the robustness study is the main role of chemotaxis. As a matter of fact, alterations of chemotaxis coefficients, especially the neutrophil ones, highly affect the behavior of the whole process, leading to a system that does not meet the basis of rules any more.

In the sensitivity study (2.2.2), we observed that no parameter influenced the final total amount of inactivated microglia. It may be explained by the fact that the mitosis rate of microglia $\left(c_{M_{i}}\right)$ is too high compared to the other terms of equation (8) As a consequence, whatever the parameter values, the final amount of inactivated microglia is always at its maximum value $\left(M_{i}=1\right)$. This behavior could be modified with the introduction of some decay for inactivated microglia. In this sensitivity study, we also noticed a quite stable behavior of adhesion molecules. If we exclude the parameters directly related to this variable, significant changes in the other parameters do not affect the behavior of these molecules in blood vessels. This sensitivity study is a first step in the exploration of the parameter space. Now with this first set of parameters we are able to manage the system in order to investigate the balance between positive and negative aspects of each components in inflammation.

After determining and studying this reference set of parameter values, we used it in the model in order to perform in silico experiments in different conditions. We studied how the size of the initial infarct could influence the development of the inflammatory process (see 2.3). The simulation results show that the aggravation due to inflammation is not linearly correlated to the infarct size, which is an interesting result. These results suggest that blocking inflammation would be more interesting for severe ischemic stroke whereas the benefit of inflammation could be stronger than the aggravation in small infarct. In this latter case, anti-inflammatory drugs could aggravate the cell damage instead of lead to beneficial effects. This point needs to be confirmed by further experiments.

We can also use this model to simulate different therapeutic strategies. Currently, the only therapeutic approach used in the Stroke Units in hospitals is thrombolysis which is aimed at reopening the obstructed vessel. We could simulate a reperfusion (i.e. a reopening of the obstructed vessel) in our model by including the role of blood flow and of ATP on each mechanism involved in the inflammatory process. Since thrombolysis can only be given to about 5 percent of stroke patients, other therapeutic strategies have been developed for about twenty years. They are called neuroprotective strategies and are aimed at blocking the biological process leading to cell death. Anti-inflammatory drugs belong to these neuroprotective approaches. The anti-inflammatory molecules already tested block either the first phase (i.e. activation of microglia) or the second phase of inflammation (i.e. infiltration of leukocytes). With our model, we can simulate different anti-inflammatory treatments acting on various targets (microglia, adhesion molecules, cytokines, neutrophils) and we can compare the effects of these treatments. Moreover, since we distinguished in the model the apoptotic cells at the beginning (reversible phase) of the apoptotic cascade and those at the end (irreversible phase) of the apoptotic cascade, we can also simulate the effects of anti-apoptotic drugs and study their roles on the ischemic damage.

The model can thus already be used to carry out in silico experiments that could contribute to a better understanding of the mechanisms involved in the inflammatory process (their influences and their connections) and of the effects of various therapeutic strategies. However, this model can also be prolonged and refined. First of all, it could be interesting to introduce in the model the rupture of the blood-brain barrier which occurs during a stroke and increases the infiltration of leukocytes in brain tissue. This could be done by adding a function that would increase membrane permeability when the density of adhesion molecules would increase (which is currently the case in the model) and when the blood-brain barrier would disrupt. Moreover, it could be interesting to add in the model a specific function describing the dynamics of endogenous anti-inflammatory cytokines. This dynamics is currently taken into account in the global function representing cytokines but it 
could be modeled in more details. This work is under development. Besides, we could also model in more details the deleterious effects of the free radicals (such as NO) that are produced by microglia and leukocytes and that can also be therapeutic targets. Finally, it could also be relevant to study the phenomenon in different geometrical conditions, for example with other distributions of vessels. Moreover, since the infarct size influences the effects of the inflammatory process, it could be interesting to perform simulations of realistic brain infarcts on a whole brain section (by adding no-flux conditions on its boundaries). To perform these simulations on brain sections, we should take into account brain geometry, brain heterogeneity (white matter / grey matter) and brain anisotropy. These features are important to study more precisely the propagation of the inflammatory process. This could also be the first step of a 3D study of the inflammatory process in ischemic stroke.

\section{REFERENCES}

[1] Christopher JL Murray and Alan D Lopez. Mortality by cause for eight regions of the world: Global burden of disease study. The Lancet, 349(9061):1269-1276, May 1997.

[2] Christopher JL Murray and Alan D Lopez. Global mortality, disability, and the contribution of risk factors: Global burden of disease study. The Lancet, 349(9063):1436-1442, May 1997.

[3] G. del Zoppo, I. Ginis, J. M. Hallenbeck, C. Iadecola, X. Wang, and G. Z. Feuerstein. Inflammation and stroke: putative role for cytokines, adhesion molecules and inos in brain response to ischemia. Brain Pathol., 10:95-112, 2000.

[4] C. Iadecola and M. Alexander. Cerebral ischemia and inflammation. Curr. Opin. Neurol., 14:89-94, 2001.

[5] K. Rupalla, P. R. Allegrini, D. Sauer, and C. Wiessner. Time course of microglia activation and apoptosis in various brain regions after permanent focal cerebral ischemia in mice. Acta Neuropathol.(Berl.), 96:172-178, 1998.

[6] M. Ankarcrona, J. M. Dypbukt, E. Bonfoco, B. Zhivotovsky, S. Orrenius, S. A. Lipton, and P. Nicotera. Glutamate-induced neuronal death: a succession of necrosis or apoptosis depending on mitochondrial function. Neuron, 15:961-973, 1995.

[7] A. Y. Lai and K. G. Todd. Differential regulation of trophic and pro-inflammatory microglial effectors is dependent on severity of neuronal injury. Glia, 56:259-270, 2008.

[8] K. Rupalla, P. R. Allegrini, D. Sauer, and C. Wiessner. Superoxide dismutase delays neuronal apoptosis: a role for reactive oxygen species in programmed neuronal death. Neuron, 14:303-315, 1995.

[9] O. Palluy and M. Rigaud. Nitric oxide induces cultured cortical neuron apoptosis. Neurosci. Lett., 208:1-4, 1996.

[10] F. Vilhardt. Microglia: phagocyte and glia cell. Int. J. Biochem. Cell Biol., 37:17-21, 2005.

[11] M. Schilling, M. Besselmann, M. Muller, J. K. Strecker, E. B. Ringelstein, and R. Kiefer. Predominant phagocytic activity of resident microglia over hematogenous macrophages following transient focal cerebral ischemia: an investigation using green fluorescent protein transgenic bone marrow chimeric mice. Exp. Neurol., 196:290-297, 2005.

[12] C. Storini, E. Rossi, V. Marrella, M. Distaso, R. Veerhuis, C. Vergani, L. Bergamaschini, and M. G. De Simoni. C1-inhibitor protects against brain ischemia-reperfusion injury via inhibition of cell recruitment and inflammation. Neurobiol. Dis., 19:10-17, 2005.

[13] J. H. Garcia, K. F. Liu, Y. Yoshida, J. Lian, S. Chen, and G. J. del Zoppo. Influx of leukocytes and platelets in an evolving brain infarct (wistar rat). Am J Pathol, 144(1):188-199, Jan 1994.

[14] J. M. Hallenbeck and A. J. Dutka. Background review and current concepts of reperfusion injury. Arch. Neurol., 47:1245-1254, 1990.

[15] A. I. Ibragimov, C. J. McNeal, L. R. Ritter, and J. R. Walton. A mathematical model of atherogenesis as an inflammatory response. Math Med Biol, 22:305-333, 2005.

[16] R. Kumar, G. Clermont, Y. Vodovotz, and C. C. Chow. The dynamics of acute inflammation. J Theor Biol, 230:145-155, 2004.

[17] D. A. Lauffenburger and C. R. Kennedy. Analysis of a lumped model for tissue inflammation dynamics. MATHEMATICAL BIOSCIENCES, 53:189-221, 1981.

[18] A. Reynolds, J. Rubin, G. Clermont, J. Day, Y. Vodovotz, and G. Bard Ermentrout. A reduced mathematical model of the acute inflammatory response: I. derivation of model and analysis of anti-inflammation. J Theor Biol, 242:220-236, 2006.

[19] R. Ladeby, M. Wirenfeldt, D. Garcia-Ovejero, L. Fenger, Dissing-Olesen, I. Dalmau, and B. Finsen. Microglial cell population dynamics in the injured adult central nervous system. Brain Res Rev, 48:196-206, 2005.

[20] A. Y. Lai and K. G. Todd. Microglia in cerebral ischemia: molecular actions and interactions. Can J Physiol Pharmacol, 84:49-59, 2006.

[21] R. H. Adrian and M. W. Marshall. Action potentials reconstructed in normal and myotonic muscle fibres. J Physiol, 258:125$143,1976$.

[22] L. Edelstein-keshet and A. Spiros. Exploring the formation of alzheimer's disease senile plaques in silico. J Theor Biol, 216:301$326,2002$. 
[23] S. M. Gray and R. Brookmeyer. Estimating a treatment effect from multidimensional longitudinal data. Biometrics, 54:976-988, 1998.

[24] M. J. Mentis, A. R. McIntosh, K. Perrine, V. Dhawan, B. Berlin, A. Feigin, C. Edwards, P. Mattis, and D. Eidelberg. Relationships among the metabolic patterns that correlate with mnemonic, visuospatial, and mood symptoms in parkinson's disease. Am J Psychiatry, 159:746-754, 2002.

[25] P. G. Ridall, A. N. Pettitt, R. D. Henderson, and P. A. McCombe. Motor unit number estimation-a bayesian approach. Biometrics, 62:1235-1250, 2006.

[26] G. Chapuisat, T. Lelekov-Boissard, M.A. Dronne, and J.P. Boissel. A detailed model of neuronal inflammation during ischemic stroke. (For a Philosophical Transaction of The Royal Society: Proceedings B), 2008.

[27] R. Eymard, T. Gallouet, and R. Herbin. Finite volume methods. Handbook of numerical analysis, 7:713-1018, 2000.

[28] M. L. Block and J. S. Hong. Microglia and inflammation-mediated neurodegeneration: multiple triggers with a common mechanism. Prog. Neurobiol., 76:77-98, 2005.

[29] S. E. Akopov, N. A. Simonian, and G. S. Grigorian. Dynamics of polymorphonuclear leukocyte accumulation in acute cerebral infarction and their correlation with brain tissue damage. Stroke., 27:1739-1743, 1996.

[30] Carmen Capone, Simona Frigerio, Stefano Fumagalli, Maurizio Gelati, Maria-Cristina Principato, Claudio Storini, Mery Montinaro, Rudolf Kraftsik, Marco De Curtis, Eugenio Parati, and Maria-Grazia De Simoni. Neurosphere-derived cells exert a neuroprotective action by changing the ischemic microenvironment. PLoS ONE, 2(4):e373, 2007.

[31] X. Wang and G. Z. Feuerstein. Induced expression of adhesion molecules following focal brain ischemia. J Neurotrauma, 12(5):825-832, Oct 1995.

[32] X. Wang, T. L. Yue, P. R. Young, F. C. Barone, and G. Z. Feuerstein. Expression of interleukin-6, c-fos, and zif268 mrnas in rat ischemic cortex. J Cereb Blood Flow Metab, 15(1):166-171, Jan 1995. 